with tincture of opium. To obviate constipation, ten or fifteen grains of the compound rhubarb pill were used; but where there was much derangement of the biliary functions, brisk purging was resorted to immediately after the cessation of a paroxysm; sufficient time being thus left for the bark being again given before the period of the next paroxysmal return. Purging was more necessary in quotidians than in the other types. Failures of the bark, which have been recorded, may be ascribed more to a want of foresight in the practitioner, than to want of virtue in the remedy. When types of the intermittent are associated with an inflammatory diathesis, the secretions generally deficient, and no measures taken to remove such before the administration of bark, the medicine may prove worse than useless; and even in the administration of quinine, a combination of it with a mercurial and James's powder, along with saline mixture and nitrous ether to act on the kidneys, will be found of great utility in the cure.

In one case of quartan, the liquor arsenicalis was given, with complete success, after mercury, oxyd of zinc, and bark, had been used in vain. The fever was of several months standing, the secretions from the liver brown and vitiated, and mercury had failed in producing free salivation. The arsenical solution was given in a dose of ten drops, immediately before the anticipated return of the paroxysm, and put an end to it at the third administration. The cessation of the fever was accompanied by a very copious salivation, and a return of healthy biliary secretion.

32 Oxford Square, London, November 1848 .

(To be concluded in next Number.)

\title{
ON THE USE OF CHLOROFORM IN SURGICAL
} OPERATIONS AND MIDWIFERY.

\section{By JOHN SNOW, M.D.}

1.-Comparison of Chloroform with Ether.-II. Circumstances which forbid its use, or modify its action.-1II. Mode of administering it. -Iv. Description of its effects.- $\nabla$. Results of its employment.vI. The cases in which Chloroform is applicuble in Miduifery.vir. Directions for its employment.

I. It is now just two years since the news of the Inhalation of Ether for preventing the pain of surgical operations, reached this country; and a little more than a year, since Chloroform was introduced as a substitute for it. The result of experience has been, to shew that either of these agents is capable of entirely preventing the pain of the most severe operations; and that when employed with care, and some little knowledge of their effects, they are free from danger. Although nothing can be effected with Chloroform which might not be accomplished by the aid of Ether, yet it possesses, as was stated by Dr. Simpson on introducing it, certain minor advantages,-in being less pungent, more portable, not leaving a disagreeable odour in the breath, and less frequently causing excitement previous to the insensibility; and these properties have been sufficient to enable it to be used, to the almost 
total exclusion of Ether from practice. There are some surgical operations, however, in which I think that Ether should still have the preference. I allude to the reduction of dislocations and strangulated herniæ, -operations in which complete relaxation of the muscles is required: as this is more easily obtained by the use of Ether. A degree of rigidity of the muscles, which gives place to a state of relaxation, by continuing the inhalation, is liable to be induced by both vapours; but is more frequent and intense, and less easily overcome, under the use of Chloroform than of Ether.

II. Any active inflammation in the head or chest ought, probably, to contra-indicate the use of Chloroform, in the present state of our knowledge respecting it ; but, as surgical operations are not undertaken, and parturition seldom occurs, under these circumstances, they can hardly be considered as limitations of its employment. Chronic affections do not appear to offer any impediment to the careful use of it. I have, on three or four occasions, administered it to patients in phthisis, requiring an operation, and several times when chronic bronchitis existed; when, so far from being injurious, it generally relieved the cough for a time. Diseases of the heart have caused more dread of danger from Chloroform than those of any other organ,-_especially since the death at Mr. Robinson's. But it appears, from the evidence adduced, that the exciting cause of the fatal event, in that instance, was rather the apprehension of what was about to be done, than the vapour which the patient had but just begun to inhale, in a very diluted state. I have seen Chloroform inhaled, without ill consequences, in several operations where the heart was more or less diseased ; and have lately been informed by a medical man, that he had administered it with relief to the difficulty of breathing attending advanced valvular disease. Consequently, if a patient with disease of the heart is obliged to undergo a painful operation, the Chloroform, so far from being probably injurious, is likely to be of the greatest service, by preventing the emotion arising both from the pain and the anticipation of it. But it should be carefully administered, so as to excite the circulation as little as possible ; and its effects carried no further, and continued no longer, than is necessary to prevent the severe part of the pain, in order to lessen the risk of subsequent sickness, and the depression which often attends it.

The general conditions of the patient, apart from acute disease, though they modify the action of Chloroform, are no obstacle to its employment or efficiency. I have had to administer it, several times, within a few weeks after birth, as well as in extreme old age, and have seen no ill effects from its use. Children are, indeed, very favourable subjects for its action, as it does not cause in them the excitement and muscular rigidity which are occasionally met with in adult patients; and immunity from pain can generally be obtained, without any thing like coma, whilst they are apparently in a dreaming state. They are quickly affected with the vapour, on account of the activity of their respiration and circulation. This should be borne in mind in practice.

Persons whose strength is somewhat reduced from any cause, also yield more quietly to the influence of Chloroform, than those in robust health. The greatest debility is no impediment to its use. Some of the patients in St. George's Hospital, who have undergone amputations, 
under both Chloroform and Ether, with a favourable result, were in such a feeble state, that, probably, the operations would not have been undertaken, except for the discovery of anæsthesia.

In patients subject to hysteria, a paroxysm is sometimes occasioned by the vapour, but it can always be quieted by continuing the inhalation.

III. Chloroform is recommended by Dr. Simpson to be given on a handkerchief : but in using such a powerful medicine as this, it is undoubtedly desirable that there should be some better means of regulating the quantity of it, in the air the patient breathes; and three cases have occurred, in different parts of the world, in which the inhalation of it, on a handkerchief, has been suddenly fatal ; apparently, from the air inspired containing so much vapour, as to impregnate the blood passing through the lungs, so strongly, that it caused paralysis of the heart, on entering that organ, and circulating through the coronary artery. There are several inhalers which permit of more or less regulation of the quantity of vapour. That which I employ, has been described in the Medical Gazette and Lancet. I never give Chloroform without the apparatus, except to keep up the insensibility during operations on the face or mouth, when it cannot be re-applied, and then I use a sponge squeezed out of cold water-putting on only ten or fifteen minims at a time.

The stomach ought not to contain much food when a patient inhales, as it would probably be vomited. The recumbent posture should be chosen when it is suitable for the operation; or if the patient must be seated, it should be on an easy chair, so that he may be duly supported, when insensible. In operations on the anus, lying on the side, with the knees drawn up, is much more convenient, as far as the Chloroform is concerned, than leaning over a table or bed, with the feet on the ground. The vapour should be much diluted at first, in order gradually to blunt the sensibility of the mucous membrane of the air-passages, and thus avoid exciting cough.

IV. To facilitate the directions for giving Chloroform, the various effects it is capable of producing on the nervous centres, may be divided into five degrees. The first degree includes the slighter effects that are experienced by the patient, whilst he retains sufficient consciousness to appreciate his situation, and know what is occurring around him. The second degree is the dreaming, or wandering state of the mind, which is observed, when the patient is not silent, immediately to follow the loss of consciousness. In the third degree, there are no voluntary movements, articulate sounds, or anything indicating the presence of ideas; but there may be involuntary muscular contractions, or rigidity. The fourth degree is a state of absolute relaxation of the voluntary muscles, in which no contraction can be excited in them. The breathing is sometimes stertorous in this degree. The fifth degree is the state of impeded respiration observed previous to death, in animals killed by Chloroform. It must be stated, that the various degrees run gradually into each other, and cannot always be clearly distinguished; and that it is seldom necessary to carry the narcotism beyond the third degree, even in the most severe operations. The pulse is generally somewhat accelerated during the inhalation, but it is not a criterion of the action of the vapour. 
The greater number of patients become quietly insensible, without offering any resistance; but, in a considerable part of them, there is some excitement, as soon as they lose their consciousness, and it is often necessary to hold their hands. A great number of female patients utter a singing sound at this stage of the inhalation. By continuing to administer the vapour the excitement is soon overcome. When voluntary motion, or talking, is no longer observed, it is desirable to examine the eye, to gain additional information as to the patient's state. If the eye be turned up, it is usually an indication that the narcotism has proceeded to the third degree, and the same is true of congestion of the ressels of the conjunctiva; but these symptoms are not met with in every case. The degree of sensibility of the conjunctiva is a better indication whether or not an operation will cause pain, than any other sign taken alone; but it is proper to take into consideration every symptom that can be observed. When the margin of the eyelid can be touched without causing contraction of the orbicularis muscle, or even when it causes but slight contraction, any operation can be performed without causing pain. As the effects of the vapour, unless very much diluted, continue to increase, for about twenty seconds, after the inhalation of it is discontinued, it is advisable, when the patient is nearly insensible, to intermit the vapour for two or three inspirations, now and then, or to dilute it with more air, if there be a valve for that purpose. Conducted in this way, the process of making the patient insensible usually occupies from two to three minutes; and this is safer than proceeding more expeditiously.

When the operation is not of very short duration, it is usually necessary to give a little Chloroform, from time to time, during its performance. Whilst important steps of an operation are in progress, it is best to prevent the least return of either voluntary motion or flinching; but in the subsidiary and concluding parts, we may wait till there is some clear sign of sensation. The loss of common sensibility generally outlasts the narcotism of the nervous centres, so that during the greater part of a protracted operation, the patient is usually in the second degree-often muttering unintelligibly, or talking in his dreams; and it now and then happens that arteries can be tied, or sutures introduced, without pain, after complete consciousness has returned. The protracted inhalation of Chloroform has a tendency to reduce the temperature of the body, consequently it is advisable to endeavour to keep the patient warm, both during the operation and afterwards; but this, of course, need not interfere with the exposure of the part that has been the seat of operation to the air, or making cold applications to it.

After the inhalation has been discontinued, the patient spontaneously recovers from its effects. Consciousness usually returns in a few minutes,-in some cases suddenly; in others, after a short period of incoherence or inebriation. It is best not to speak to the patient prematurely, but to quietly await the complete return of consciousness.

v. The patient, after a severe operation under Chloroform, is in a calmer and more cheerful state of mind than if he had suffered the pain; and after an amputation he seldom experiences nervous starting of the stump. These are circumstances that cannot fail to contribute to his 
recovery; and as far as statistics of the larger operations have been collected, they are favourable to the practice of anæsthesia.

vi. The objections which have been made to the use of Chloroform in midwifery are nearly all $a$ priori ones, and are capable of being met by replies of the same nature; but a question, concerning a line of medical practice, should be examined and decided on chiefly by experience. There are many labours so favourable, that the patient bears what pain there is cheerfully, and makes no complaint, unless it be for a few moments, whilst the child's head is passing through the external parts. In cases of this kind, which are probably the truly healthy ones, Chloroform does not seem to be indicated, and $I$ have not seen it applied. The cases in which I have administered it, have been protracted, or attended with severe pain, or both these conditions have been combined; or they have been cases in which manual or instrumental aid has been required, and it has always afforded the greatest relief, and been attended with no ill effects, to the mother or her offspring. There was hæmorrhage after the birth of the child in one instance, but the patient had suffered from the same occurrence in previous confinements.

vIr. The dose of vapour required at one time, in unassisted labour, is very much less than for a surgical operation. It is only to stop strong uterine action, in order to facilitate turning the child, that a full chirurgical dose of the vapour is required in obstetric practice; for, in instrumental delivery, there is less pain, and less necessity for the patient to be perfectly still, than in operations in which the knife is used. Chloroform should be given in midwifery, as in surgical operations, with an apparatus; or if the medical attendant be unprovided with one, he should put only from ten to fifteen minims on the handkerchief at a time. I feel obliged entirely to dissent from the practice recommended by Dr. Simpson, in the Monthly Journal of Medical Science for October last, of putting three or four drachms on the handkerchief to begin with. I do not dispute that he has tact enough to practice this plan without accident, but I feel sure that it will lead to fatal results in other hands. I also disapprove of his practice of handing over the handkerchief afterwards to the husband or nurse. This method of administering Chloroform is also objectionable from the quantity of vapour that becomes diffused through the air of the room; and from Dr. Simpson's account of the quantity employed, it must cost a shilling an hour more than the plan I employ. This I mention, merely to show that dispensing with the use of an apparatus is not a saving.

When the first stage of labour is tedious and painful, and the patient wearied and desponding, the greatest relief may often be afforded without altogether removing consciousness ; and whilst the uterine contractions are not powerful, it is best to give but a very small quantity of Chloroform, or they become suspended, which, however, is of no great consequence, as they soon return. The best way of administering the vapour, is to let the patient inhale a little at the beginning of each pain. Commencing with the inhalation early in labour, does not entail a necessity of continuing it throughout; for the patient, knowing that she can have occasional relief, often bears the pain more cheerfully. And after the Chloroform is discontinued, it exerts sufficient influence, for a time, to counteract the persistent uneasiness there often is between 
the pains, and thus to enable the patient to have short periods of refreshing sleep.

When the pains are stronger, rather more Chloroform can be given, without retarding the progress of the labour, but the effect of it should not exceed the second degree; and except in obstetric operations, the usual symptoms of labour ought to continue. The object should be only to prevent the patient's sufferings by making her unconscious; and whilst articulate complaints and loud cries are prevented, to allow the reflex or instinctive auxiliary action of the respiratory, and even voluntary muscles to continue, accompanied, perhaps, with some moaning. I believe that, with a little management, Chloroform may generally be administered without retarding the labour; and in cases rendered tedious by rigidity of the os uteri, or obstructed by an unyielding state of the perineum, Chloroform shortens the duration of labour very much, by causing the relaxation of these parts. There are instances, also, in which a moderate exhibition of the vapour seems to strengthen the reflex bearing-down efforts, which had probably been diminished by fear of the pain.

Chloroform has been given with advantage by Mr. Landsdowne, of Bristol, and others, for the relief of severe after-pains. It may be used for this purpose in some cases where it has not been required during labour.

Losdon, Frith Street, Soho Square, December 1818.

\section{AN ANOMALOUS CASE OF SPINAL AFFECTION.}

By GOLding BIRD, A.M., M.D., F.R S., Fellow of the Royal College of Physicians; Assistant.Physician to, and Professor of Materia Medica at, Guy's Hospital.

[THE following notice of a very remarkable case was read before the Physical Society of Guy's Hospital three yeurs ago. Its, to me, unique character, has induced me to think it worthy of publication. The only alteration made in the original paper, has been the somewhat abridging of it.-G. B.]

No one can for any length of time be engaged in the active practice of his profession, or in the observation of disease, without encountering phenomena sufficiently puzzling to surround him with unforeseen difficulties, and often sufficiently inexplicable to force upon his mind the conclusion, that medical science, or at least the share of it falling to his lot, is by no means perfect or exact. I believe this will be granted by all whose experience has been extensive enough to produce humility; for a sense of perfection in acquirements generally goes hand in hand with limited knowledge. Although I do not assent to the Celsian dogma, Medicina est ars conjecturalis, still cases like the one $I$ hope to make the text for a profitablediscussion this evening, show us, valueless as are mere conjectural arguments, how carefully we must go on collecting instances, (in the phraseology of Bacon), before we can hope to draw inductions sufficiently extended to act as the keys to the obscurity shrouding the causes of many pathological conditions. 